\title{
Pituitary gland volume in at-risk mental state for psychosis: a longitudinal MRI analysis
}

Anna Walter $^{\mathrm{a}}$, MD; Erich Studerus ${ }^{\mathrm{b}}, \mathrm{PhD}$; Renata Smieskova ${ }^{\mathrm{a}, \mathrm{c}}, \mathrm{PhD}$; Corinne Tamagni ${ }^{\mathrm{b}}$,

PhD; Charlotte Rapp ${ }^{\mathrm{b}}, \mathrm{PhD}$; Stefan J. Borgwardt ${ }^{\mathrm{a}, \mathrm{c}}$, MD PhD; Anita Riecher-Rössler ${ }^{\mathrm{b} *}, \mathrm{MD}$

$\mathrm{PhD}$

${ }^{\text {a }}$ University of Basel Psychiatric Clinics, Wilhelm Klein-Strasse 27, CH-4012 Basel,

Switzerland

${ }^{\mathrm{b}}$ University of Basel Psychiatric Clinics, Center for Gender Research and Early Detection, Kornhausgasse 7, CH-4051 Basel, Switzerland

${ }^{\mathrm{c}}$ Medical Image Analysis Centre (MIAC), University Hospital Basel, Schanzenstrasse 55, CH-4031 Basel, Switzerland

\section{*Corresponding Author:}

Prof. Anita Riecher-Rössler, MD

University of Basel Psychiatric Clinics

Center for Gender Research and Early Recognition

Kornhausgasse 7

CH-4051 Basel, Switzerland

Phone: +41613258161

Fax: +41613258160

E-mail: anita.riecher@upkbs.ch

\section{Authors' e-mail addresses:}

anna.walter@upkbs.ch; erich.studerus@upkbs.ch; renata.smieskova@upkbs.ch;

corinne.tamagni@gmail.com; charlotte_rapp@hotmail.com; stefan.borgwardt@upkbs.ch

\section{Acknowledgements}

We would like to thank all patients who participated in the study as well as Claudine Pfister and Laura Egloff for their help in preparing and editing this manuscript. 


\begin{abstract}
Introduction: Pituitary enlargement has been reported in individuals with schizophrenic psychosis or an at-risk mental state for psychosis (ARMS). In a previous study, our group could show pituitary volume increase in first episode and ARMS patients with later transition to psychosis (ARMS-T). However, there are no longitudinal studies on this issue so far. We therefore examined longitudinally whether transition to psychosis would be accompanied by a further increase of pituitary volume in antipsychotic-naïve ARMS patients.
\end{abstract}

Methods: Magnetic resonance imaging (MRI) data were acquired from 23 antipsychoticnaïve individuals with an ARMS. Ten subjects developed psychosis (ARMS-T) and 13 did not (ARMS-NT). ARMS-T were re-scanned after the onset of psychosis, ARMS-NT at the end of the study period.

Results: There was no significant difference of the pituitary volume between ARMS-T and ARMS-NT in our sample and there were no significant pituitary volume changes over time.

Discussion: Longitudinally, we could not detect any further volumetric changes in the pituitary volume with transition to psychosis.

Conclusions: This, together with the result of our previous study, could indicate that the perceived level of stress in ARMS patients is constantly high from very early onwards.

Keywords: schizophrenic psychoses; hypothalamic-pituitary-adrenal axis (HPA-axis); stress; prolactin; ACTH; neuroimaging 


\section{Introduction}

Enlargement of the pituitary gland has been reported in schizophrenic psychoses and patients with an at-risk mental state for psychoses (ARMS). ${ }^{1}$

Cross-sectional studies indicate that pituitary volume (PV) is increased at psychosis onset ${ }^{2}$ and even in the prodromal stage of the disease. ${ }^{1,3}$ This enlargement seems to be more pronounced in ARMS with later transition to psychosis compared to ARMS without transition to psychosis. ${ }^{3} \mathrm{PV}$ seems to be further increased by most antipsychotics ${ }^{4}$ through lactotroph hyperplasia. $^{5}$

Studies with chronic schizophrenia patients suggest that initial enlargement is followed by a decrease of PV., ${ }^{4,6}$ The decrease following the initial enlargement has not only been found in treated ${ }^{4}$ but also in untreated ${ }^{7}$ patients and might be caused amongst others by a chronic activation of the HPA axis. ${ }^{4,8}$

Results of longitudinal MRI studies are less consistent than those of cross-sectional studies due to different types of medications at baseline and during follow-up, as well as to the length of the follow-up. To our knowledge, only four longitudinal MRI studies on PV changes in first episode psychosis (FEP) patients have been conducted. MacMaster et al. ${ }^{9}$ found a $12 \%$ increase in an originally antipsychotic-naïve population with schizophrenia, possibly driven by the prolactin-elevating antipsychotic risperidone (follow-up period 12 months). Takahashi et al. ${ }^{10}$ also found an enlargement over time in 18 FEP and 13 schizotypical disorder patients (all on antipsychotic treatment at baseline and follow-up) over a follow-up period of approximately 2.7 years. The third study ${ }^{11}$ showed a dose-dependent reduction of PV in patients with atypical antipsychotic medication during a 3-months follow-up. As in this study mostly quetiapine was used, which does not induce hyperprolactinemia, this might reflect the "natural course" of PV development. A recently published study in 55 FEP patients did not find changes in PV following antipsychotic treatment. ${ }^{12}$ 
However, to date, there are no longitudinal studies on PV changes in ARMS individuals.

Hence, this study aimed to elucidate how PV changes develop during emerging psychosis. Specifically, we aimed to investigate PV changes associated with the onset of psychosis by comparing subjects with an ARMS with later transition to psychosis (ARMS-T) versus those without transition (ARMS-NT). In longitudinal analysis, we expected to find a progression of PV enlargement in ARMS-T compared to ARMS-NT as stress on the one hand is known to trigger the transition to psychosis and on the other hand to increase PV due to activation of ACTH and prolactin synthesis. ${ }^{3,6,13-17}$

\section{Methods}

\section{Study design}

This imaging study was embedded in the Früherkennung von Psychosen (FePsy) study which is an open, prospective clinical study of all consecutive referrals to a specialized clinic for the early detection of psychosis. ${ }^{18,19}$ From March 1, 2000, to February 29, 2004, individuals with suspected ARMS were identified and investigated in various modalities, including psychopathology and neuropsychology. Follow-up assessments of all ARMS individuals were conducted at regular intervals (see below).

\section{Setting}

Subjects were recruited through the FePsy Early Detection Clinic at the University Psychiatric Outpatient Department, Basel, Switzerland, a clinic specifically designed to identify ARMS. To encourage early, low-threshold referrals, widespread information campaigns targeting potential referring professionals and lay people were conducted. ${ }^{18}$ All referrals to the clinic during the study period were screened as described below. 
The study was described comprehensively to the subjects and written informed consent was obtained as per approved guidelines from the University of Basel local ethics committee $(\mathrm{EKBB})$.

\section{Inclusion criteria}

Inclusion as ARMS required one or more of the following: (a) "attenuated" psychotic symptoms (scores of 2 or 3 on the hallucination item, 3 or 4 on the unusual thought content or 3 or 4 on the suspiciousness items of the $\mathrm{BPRS}^{20}$ for at least several times a week and persisting for more than 1 week), (b) brief limited intermittent psychotic symptoms (BLIPS) (scores of 4 or above on the hallucination item, or 5 or above on the unusual thought content, suspiciousness or conceptual disorganization items of the BPRS, with each symptom lasting less than 1 week before resolving spontaneously), (c) a first degree relative with a psychotic disorder plus at least two indicators of a clinical change, such as marked decline in social or occupational functioning, or (d) minimal amount and combination of unspecific risk factors according to the Basel Screening Instrument for Psychosis (BSIP) $)^{21}$ and proficiency in the German language.

\section{Exclusion criteria}

These were: age below 18 years, insufficient knowledge of German, IQ < 70, previous episode of diagnosed and treated schizophrenic psychosis or treatment with antipsychotics for more than 3 weeks (lifetime) equivalent to a chlorpromazine dose of $2500 \mathrm{mg},{ }^{22}$ psychosis due to organic brain disease including substance dependency, or psychotic symptoms within a clearly diagnosed affective disorder or borderline personality disorder. For this part of the study only patients with normal thyroid function were included. When it was not suitable to perform an MRI (e.g. pacemaker, metal in the body), subjects were excluded from the imaging part of the FePsy study. 


\section{Measures}

\section{Screening Instrument}

The ARMS was assessed using the BSIP, which was specifically developed for this purpose. ${ }^{21}$ The BSIP is based on the DSM-III-R prodromal symptoms and other prodromes as derived from literature as well as social decline, drug abuse, previous psychiatric disorders, and genetic risk; four psychosis items of the expanded version of the BPRS ${ }^{20}$ for assessing (pre-) psychotic phenomena were incorporated. The BSIP allows the rating of individuals regarding inclusion/exclusion criteria corresponding to the PACE (Personal Assessment and Crisis Evaluation) criteria ${ }^{23,24}$ and has been shown to have a good inter-rater reliability for the assessment of the main outcome category "at risk for psychosis" and a high predictive validity. ${ }^{21}$

\section{Entry Examination}

All subjects selected for the study by screening subsequently underwent an extensive entry examination, which included the BPRS and the Scale for the Assessment of Negative Symptoms (SANS). ${ }^{25}$

\section{Follow-Up}

Each ARMS individual was followed up at regular intervals. During the first year of followup, high-risk individuals were assessed monthly using BPRS and SANS. During the second and third years, all individuals were assessed every 3 months and thereafter once annually. Transition to psychosis was monitored, applying the transition criteria of Yung et al. ${ }^{23}$

\section{Subjects}

For this study, 23 antipsychotic-naïve individuals with an ARMS were included. Applying the above mentioned transition criteria, 10 of the 23 ARMS individuals made the transition to 
psychosis (ARMS-T) during follow-up and 13 did not transition to psychosis during the follow-up period (ARMS-NT). The relatively high transition rate (43\%) is explained by the method we chose the patients. Of our original sample of ARMS patients ${ }^{3}$ we chose the first 10 patients with transition to psychosis (ARMS-T) and invited them for a follow-up scan. The group of ARMS-NT was then matched to the group of ARMS-T in terms of age, gender, and education. Mean follow-up periods were 1034 and 1689 days in ARMS-T and ARMS-NT, respectively.

Baseline MRIs are part of our cross-sectional hippocampal volume study ${ }^{26}$ and the sample partly overlaps with the sample of our longitudinal hippocampal volume study in ARMS. ${ }^{27}$

\section{Image acquisition}

Subjects were scanned at the time of study inclusion and were re-scanned after the onset of psychosis or at the end of the follow-up (approximately 5 years after inclusion to the study) if they did not develop psychosis.

All patients at baseline had $1.5 \mathrm{~T}$ scanning, part of the follow-up scans were done with the $3 \mathrm{~T}$ scanner. Head movement was minimized by foam padding and Velcro straps across the forehead and chin. A three-dimensional volumetric spoiled gradient recalled echo sequence generated 176 contiguous, 1-mm thick sagittal slices. Imaging parameters were: time-to-echo, 3.04-4 ms; time-to-repetition, 1.9-9.7 ms; flip angle, 12-15; matrix size, $200 \times 256$; field of view, $25.6 \times 25.6 \mathrm{~cm}$ matrix; voxel dimensions, $1 \times 1 \times 1 \mathrm{~mm}$ for the $1.5 \mathrm{~T}$ scanner, respectively: time-to-echo, $3.37 \mathrm{~ms}$; time-to-repetition, $2 \mathrm{~ms}$; flip angle, 8 ; matrix size, $256 \times$ 256; field of view, $25.6 \times 25.6 \mathrm{~cm}$ matrix; voxel dimension, $1 \times 1 \times 1 \mathrm{~mm}$ for the $3 \mathrm{~T}$ scanner.

\section{Image analysis}


Original DICOM data were then converted to Analyze 7.5 format in axial direction coded to ensure blindness of the investigator and patients' confidentiality and finally imported into the image-analysis software AMIRA. ${ }^{28}$

The pituitary glands were manually traced in the interactive public domain software program AMIRA using a method described by Sassi et al. ${ }^{8}$ The pituitary stalk was excluded, but the posterior pituitary was included. We traced around the borders of the pituitary in the same way as previously described: ${ }^{1,4,9,11}$ the diaphragma sellae superiorly, the sphenoid sinus inferiorly, and the cavernosus sinus laterally. First, the pituitary was traced in all coronal slices where it could be visualized. In addition to the method of Sassi et al., ${ }^{8}$ the tracing was then corrected in sagittal and axial dimensions to be finally controlled on the coronal slices again. The software calculates the traced ROI volume in $\mathrm{mm}^{3}$ by summing the number of voxels of each traced slice.

All measurements were carried out by a trained and blinded rater (AW). The intra-rater intraclass correlation coefficient in a subset of 10 randomly selected brains was 0.81 . All of our baseline scans have been part of the sample published in a cross-sectional investigation of our group $^{3}$. Inter-rater reliability was estimated based on 10 randomly selected cases measured in our previously published study ${ }^{3}$ and remeasured now by the first author of this paper. The inter-rater intra-class correlation was 0.718 .

The whole brain volume was measured to correct for differences in head size as previously described. $^{27,29-31}$

\section{Statistical analysis}

Analyses were performed using the $\mathrm{R}$ environment for statistical computing. ${ }^{32}$ Clinical and demographic differences between groups were examined with Fisher's Exact test and Welch Two Sample t-test. PV changes over time were analyzed by using a linear mixed effects model that included a random intercept factor for the subjects and fixed effects for time, 
group (ARMS-T vs. ARMS-NT), age, gender, medication, whole brain volume (baseline), scanner, and time by group and time by medication interactions. $P$-values of the fixed effects parameters were estimated by using Markov Chain Monte Carlo (MCMC) simulations using the pvals.fnc function in the languageR package. ${ }^{33}$

\section{Results}

\section{Demographic and clinical data}

Regarding sex, age, education, and current cannabis use at baseline, there were no significant differences between ARMS with subsequent transition and ARMS without later transition to psychosis. However, there was a significantly higher SANS score and a trend towards higher BPRS scores already at baseline in ARMS-T compared to ARMS-NT (Table 1). All patients were antipsychotic-naïve at baseline, but 6 of the ARMS-T patients were treated with antipsychotics ( 2 olanzapine, 1 risperidone, 1 amisulpride, 1 perphenazine) at the second scan. This can be explained by the fact that these patients at transition often had to be treated before they could be scanned. The average time between transition and second scan was 802 days. All ARMS-NT subjects were still antipsychotic-naïve at the follow-up scan. Compared to ARMS-T, the inter-scan (t0 - tx) interval was slightly longer in ARMS-NT ( $p=0.015)$ as we wanted to be sure not to miss a transition.

\section{Pituitary volume}

The mixed effects model revealed significant main effects of sex $(\mathrm{p} \leq 0.001)$, and medication $(\mathrm{p}=0.005)$ : PVs were significantly larger in women and in patients treated with antipsychotics at the second scan. 
We found no significant main effects of group, age, whole brain volume, scanner, and time by group and time by medication interactions. Thus, PV did not change over time in neither group when corrected for confounders as described above (i.e. including medication).

Whole brain volume was significantly smaller in women than in men (Table 2), but not significantly different between ARMS-T and ARMS-NT. We also found no significant association of whole brain volume with PV $(\mathrm{p}=0.103692)$, and the above mentioned results did not change whether we considered whole brain volume or not.

\section{Correlational analyses}

In a next step, we tested whether PV was associated with the severity of psychopathological symptoms. PV did not significantly correlate with BPRS total score $(r=0.1, t=0.44, \mathrm{df}=18$, $\mathrm{p}=0.662)$ or SANS total score $(\mathrm{r}=0.12, \mathrm{t}=0.51, \mathrm{df}=18, \mathrm{p}=0.61)$, even if we only included ARMS-T $(r=-0.06, t=-0.16, \mathrm{df}=8, \mathrm{p}$-value $=0.874$ and $\mathrm{r}=0.07, \mathrm{t}=0.21, \mathrm{df}=8, \mathrm{p}$-value $=$ 0.838 , respectively).

\section{Discussion}

To our knowledge, this is the first longitudinal study investigating PV in an antipsychoticnaïve ARMS population comparing ARMS with subsequent transition to psychosis versus ARMS without transition.

We studied 23 antipsychotic naïve individuals with an ARMS within the FePsy study at baseline. Patients were followed up until transition to psychosis (ARMS-T) or, if they did not develop psychosis (AMRS-NT), until the end of the study.

In contrast to our hypothesis, we did not find a time dependent longitudinal PV increase in the ARMS-T group from baseline to transition. Cross-sectionally we had shown a stronger PV enlargement in ARMS with later transition to psychosis than in ARMS without later transition 
and compared to healthy controls (HC) in a previous study of our group ${ }^{3}$. The sample partly overlaps, with the one presented here. A recently published cross-sectional study found no difference between ARMS with and without later transition to psychosis. ${ }^{34}$ We had hypothesized that the enlargement at baseline was due to stress-induced ACTH and prolactin synthesis. Based on our current finding of no further PV increase during actual transition to psychosis one might conclude that the perceived level of stress in ARMS patients is already high at first presentation to special early detection clinic and remains constantly high thereafter. As we have no objective measures of stress in our study this remains speculative.

Alternatively, the lack of a further longitudinal PV increase might be due to methodological limitations: due to the long period (on average $802( \pm 722)$ days) between transition to psychosis and follow-up scan in ARMS-T, we might have missed the expected additional stress-induced peak of PV on the time point of transition. A decrease of PV during the later course of schizophrenic psychoses has been described before in medicated ${ }^{4,6}$ and unmedicated $^{7}$ patients.

Currently, the stress-vulnerability model is commonly accepted in psychotic disorders. ${ }^{35-37}$ The literature provides some evidence that the onset of psychotic disorders may be associated with a higher rate of stress. One biological system triggered by stress exposure is the hypothalamic-pituitary-adrenal (HPA) axis. There is evidence for dysfunction of this system in schizophrenic diseases. ${ }^{15,38-42}$ We assume that pituitary volume is a structural means of its activity in patients with psychosis. ${ }^{11}$ Indeed, pituitary volume has been used as an indirect index of HPA axis activity because it is thought to increase with the number or size of corticotrophin-releasing cells (i.e., corticotrophs) in the pituitary gland. ${ }^{1,43-45}$ Volumetric abnormalities of the pituitary gland in psychotic disorders strengthen this theory. ${ }^{46}$ Nonetheless, there are some contradictory results, ${ }^{11}$ but most studies showing smaller PVs were conducted in patients with a chronic type of schizophrenic psychosis. ${ }^{4,7,47}$ 
We hypothesize that prolactin as a stress hormone can be involved in the increase of PV, but it remains unclear what is its cause and its consequence. Previously, PV enlargement in schizophrenic psychoses has only been associated with prolactin-increasing antipsychotics., ${ }^{2,9}$ However, even before antipsychotics were discovered, gonadal dysfunction in schizophrenic patients has been reported; for review see Riecher-Rössler \& Häfner $1993 .^{48}$ This fact could indicate that hyperprolactinemia, which suppresses gonadal function, could be a stressinduced phenomenon in schizophrenic psychoses which is independent of antipsychotics. $^{3,14,49-51}$ As prolactin is produced in the lactotroph cells of the pituitary gland, this could also explain PV increase in unmedicated ARMS individuals. Hyperprolactinemia in antipsychotic-naïve ARMS individuals ${ }^{14}$ and first episode psychosis patients ${ }^{13,14,52,53}$ has been shown before.

There are only four longitudinal studies on PV in patients with schizophrenic psychoses, ${ }^{9,11,12,54}$ but none in ARMS individuals and none with a follow-up period of more than 2.7 years. The strength of our study is that we did the first longitudinal study of PV in ARMS. Also, all patients at baseline were unmedicated, and the follow-up period was very long, so we can be quite sure not to have missed a transition to psychosis.

PVs were significantly larger in patients who were treated with antipsychotics at time of follow-up; this result is consistent with previous results. ${ }^{4,5}$ It has been assumed that the pituitary gland is especially sensitive to prolactin-elevating antipsychotics. ${ }^{9,55}$ There are also results showing that "atypical" antipsychotics - not further differentiated - correlate with a less severe pituitary enlargement. ${ }^{10}$ We have controlled statistically for the influence of antipsychotic intake. However, our sample was too small to differentiate between different types of antipsychotics. Other causes of pituitary enlargement, such as hypothalamic tumor, pregnancy, and primary hypothyroidism ${ }^{56}$ were excluded in our sample. 
In this study women had larger PVs than men. These findings are consistent with previous reports of sex differences in PV, with PVs being larger in healthy women than in men. ${ }^{57-59}$ According to the stress hypothesis, we would have expected a positive relationship between a higher BPRS or SANS total score, implying a higher level of stress to be associated with increased PV. However, we did not find significant correlations between clinical symptoms (BPRS and SANS) and pituitary volume, which has previously been described also by others. ${ }^{9,45}$ In our case, this might be because we measured BPRS and SANS only at baseline and psychopathology can change rapidly.

Many previous studies, ${ }^{6,9,60,61}$ but not all, ${ }^{62}$ have provided evidence that pituitary volume reflects hormonal activity. Hence, we chose a volumetric approach to evaluate the hormonal function. Other studies have provided no evidence for a relation between PV and current basal levels of anterior pituitary hormones. ${ }^{62}$ We did not measure hormone levels at the time of scan acquisition and therefore do not know if HPA and HPG activity at the time of MRI was really abnormal. But we know from another study in our FePsy sample that $24 \%$ of our antipsychotic-naïve ARMS patients and 33\% of our antipsychotic-naïve FEP patients suffered from hyperprolactinemia. ${ }^{14}$

In line with other recent studies, ${ }^{46,63}$ we did not distinguish between the anterior and posterior part of the pituitary. This might be seen as a potential limitation of our study, but the posterior part of the pituitary constitutes only a very small part of the whole gland and no conditions are known to influence it in the examined age group. ${ }^{43}$ Thus, it has been hypothesized that the whole pituitary volume is representative for the anterior lobe. ${ }^{64}$

Another limitation might be that the cycle state of the women included in our study was not taken into account. However, a recent study has shown that cycle phase, number of given births, and onset or duration of menopause have no effects on pituitary size. ${ }^{61}$ 
Last but not least, small effects might have been overlooked due to our obviously small sample size and limited power. In order to estimate the likelihood of a type II error, we would have to conduct a formal power analysis. However, since there are no longitudinal studies on pituitary volume changes in ARMS patients, let alone meta-analyses, we cannot make a reliable assumption about the pituitary volume change in the population of ARMS patients in a specific amount of time and thus are unable to do a proper power analysis. Doing a post hoc power analysis by taking the effect size that we have measured in our own sample as a population effect size estimate would be very inappropriate and has strongly been criticized. ${ }^{65}$ Hopefully, our study could still contribute to the detection of small effects in the future if data will be included into meta-analyses.

Further studies should include: anatomical scanning at the time transition, additional measurements of hormones (prolactin, cortisol), comparisons with other diseases, a healthy control group with longitudinal measurements, and larger samples.

\section{Conclusions}

Pituitary enlargement has been reported in individuals with schizophrenic psychosis or an atrisk mental state for psychosis (ARMS). To our knowledge, this is the first longitudinal study investigating PV in an antipsychotic-naïve ARMS population comparing ARMS with subsequent transition to psychosis versus ARMS without transition. Only partly supporting our hypothesis, we did not find a time-dependent PV increase in the ARMS-T group from baseline to transition. This might indicate that the perceived level of stress in ARMS patients is constantly high from very early onwards. Further studies should include: anatomical scanning at the time transition, additional measurements of hormones (prolactin, cortisol), comparisons with other diseases, a healthy control group with longitudinal measurements, and larger samples. 


\section{Disclosures}

SB reports having received lecture fees from Eli Lilly; and research funding from the University of Basel, the EU, and the Swiss National Science Foundation Foundation (grant number 3232BO_119382).

ARR reports having received research funding from the Foundation Alamaya, the „Österreichische Forschungsförderungsgesellschaft mbH, Wien", the EU, the Stanley Foundation (grant number \#07TGF-1102), the Swiss National Science Foundation (grant numbers 320033_1275443/1; 320030_127323/1), the Bruderer-Stiftung.

RS reports having received funding from the Swiss National Science Foundation (grant number 3232BO_119382).

The other authors report no potential conflict of interest. 
TABLE 1. Sociodemographic and clinical sample description

\begin{tabular}{|c|c|c|c|}
\hline & $\begin{array}{l}\text { ARMS-NT } \\
(n=13)\end{array}$ & $\begin{array}{l}\text { ARMS-T } \\
(n=10)\end{array}$ & $\begin{array}{l}\text { Group } \\
\text { comparisons }\end{array}$ \\
\hline \multicolumn{4}{|l|}{ Sex } \\
\hline Women & $\mathrm{n}=5(38 \%)$ & $\mathrm{n}=3(30 \%)$ & $\mathrm{p}=1^{1}$ \\
\hline Men & $\mathrm{n}=8(62 \%)$ & $\mathrm{n}=7(70 \%)$ & \\
\hline Age at baseline scan, mean [years] (SD) & $23.1(5.7)$ & $25.3(6.7)$ & $p=0.43^{2}$ \\
\hline Education, mean [years] (SD) & $10.8(3.3)$ & $10.80(1.6)$ & $p=0.99^{2}$ \\
\hline BPRS total score at baseline & $37.1(6.9)$ & $43.8(8.7)$ & $p=0.077^{2}$ \\
\hline SANS total score at baseline & $16.4(11.5)$ & $32.9(20.4)$ & $p=0.049^{2}$ \\
\hline Antipsychotic intake at baseline scan & $0 \%$ & $0 \%$ & \\
\hline Antipsychotic intake at 2 nd scan & $\mathrm{n}=0(0 \%)$ & $\mathrm{n}=6(60 \%)$ & $p=0.002^{1}$ \\
\hline Inter-scan interval t0-tx, mean [days] (SD) & $1689(438)$ & $1034(648)$ & $p=0.015^{2}$ \\
\hline $\begin{array}{l}\text { Time between transition and } 2^{\text {nd }} \text { scan, mean } \\
\text { [days] (SD) }\end{array}$ & - & $802(722)$ & \\
\hline Cannabis use at baseline & & & $p=0.28$ \\
\hline None & $53 \%$ & $75 \%$ & \\
\hline Rarely & $7 \%$ & $12 \%$ & \\
\hline Several times per month & $7 \%$ & $0 \%$ & \\
\hline Several times per week & $13 \%$ & $0 \%$ & \\
\hline Daily & $20 \%$ & $12 \%$ & \\
\hline
\end{tabular}


TABLE 2. Pituitary and whole brain volume by group

\begin{tabular}{llll}
\hline & ARMS-NT & ARMS-T & Group comparison \\
& $\mathrm{n}=13$ & $\mathrm{n}=10$ & \\
\hline PV at $1^{\text {st }}$ scan & $0.540(0.055)$ & $0.581(0.110)$ & $\mathrm{t}=-0.9, \mathrm{p}=0.3^{1}$ \\
PV at $2^{\text {nd }}$ scan & $0.573(0.094)$ & $0.617(0.046)$ & $\mathrm{t}=-0.2, \mathrm{p}=0.42^{1}$ \\
WBV at $^{\text {st }}$ scan & $1313(116)$ & $1299(114)$ & $\mathrm{t}=4.1, \mathrm{p}=0.78^{1}$ \\
\hline
\end{tabular}

Data are presented as mean [ml] (SD); $P V=$ pituitary volume; $\mathrm{WBV}=$ whole brain volume

${ }^{1}$ Welch Two Sample t test 
TABLE 3. Gender differences. Pituitary and whole brain volume by group

\begin{tabular}{lllll}
\hline & & Women & Men & Group comparison \\
\hline ARMS-NT & PV at $1^{\text {st }}$ scan & $0.58(0.04)(\mathrm{n}=5)$ & $0.51(0.04)(\mathrm{n}=8)$ & $\mathrm{t}=3.1, \mathrm{p}=0.012^{1}$ \\
& PV at $2^{\text {nd }}$ scan & $0.65(0.03)$ & $0.52(0.09)$ & $\mathrm{t}=3.8, \mathrm{p}=0.004^{1}$ \\
& WBV at $1^{\text {st }}$ scan & $1221(99)$ & $1371(87)$ & $\mathrm{t}=-2.8, \mathrm{p}=0.025^{1}$ \\
ARMS-T & PV at $1^{\text {st }}$ scan & $0.72(0.04)(\mathrm{n}=3)$ & $0.52(0.07)(\mathrm{n}=7)$ & $\mathrm{t}=5.4, \mathrm{p}=0.001^{1}$ \\
& PV at $2^{\text {nd }}$ scan & $0.79(0.09)$ & $0.54(0.09)$ & $\mathrm{t}=4, \mathrm{p}=0.022^{1}$ \\
& WBV at $1^{\text {st }}$ scan & $1181(81)$ & $1350(85)$ & $\mathrm{t}=-3, \mathrm{p}=0.039^{1}$
\end{tabular}

Data are presented as mean [ml] (SD); PV = pituitary volume; $\mathrm{WBV}=$ whole brain volume

${ }^{1}$ Welch Two Sample t test 


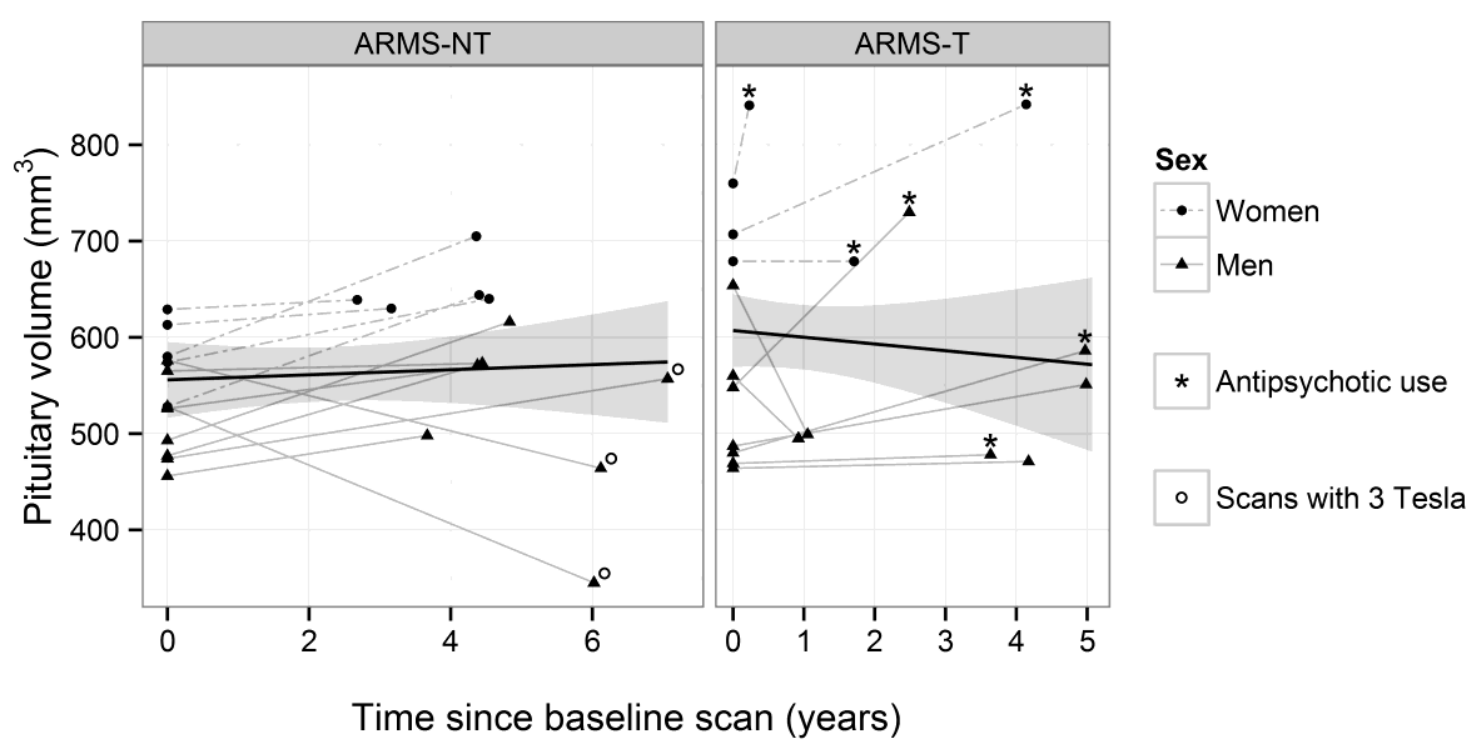

FIGURE 1. Volume changes of the pituitary gland in patients with an ARMS without transition (ARMS-NT) and with transition to psychosis (ARMS-T). Values of baseline and follow-up scans in each subject are connected with a straight line. 


\section{References}

1. Garner B, Pariante CM, Wood SJ, et al. Pituitary volume predicts future transition to psychosis in individuals at ultra-high risk of developing psychosis. Biol Psychiatry. Sep 1 2005;58(5):417-423.

2. Pariante $\mathrm{CM}$, Dazzan $\mathrm{P}$, Danese A, et al. Increased pituitary volume in antipsychotic-free and antipsychotic-treated patients of the AEsop first-onset psychosis study. Neuropsychopharmacology. Oct 2005;30(10):1923-1931.

3. Büschlen J, Berger GE, Borgwardt SJ, et al. Pituitary volume increase during emerging psychosis. Schizophr Res. Jan 2011;125(1):41-48.

4. Pariante $\mathrm{CM}$, Vassilopoulou $\mathrm{K}$, Velakoulis $\mathrm{D}$, et al. Pituitary volume in psychosis. $\mathrm{Br} \mathrm{J}$ Psychiatry. Jul 2004;185:5-10.

5. Markianos M, Hatzimanolis J, Lykouras L. Neuroendocrine responsivities of the pituitary dopamine system in male schizophrenic patients during treatment with clozapine, olanzapine, risperidone, sulpiride, or haloperidol. Eur Arch Psychiatry Clin Neurosci. Jun 2001;251(3):141-146.

6. Pariante CM. Pituitary volume in psychosis: the first review of the evidence. $J$ Psychopharmacol. Mar 2008;22(2 Suppl):76-81.

7. Upadhyaya AR, El-Sheikh R, MacMaster FP, Diwadkar VA, Keshavan MS. Pituitary volume in neuroleptic-naive schizophrenia: a structural MRI study. Schizophr Res. Feb 2007;90(13):266-273.

8. Sassi RB, Nicoletti M, Brambilla P, et al. Decreased pituitary volume in patients with bipolar disorder. Biol Psychiatry. Aug 15 2001;50(4):271-280.

9. MacMaster FP, El-Sheikh R, Upadhyaya AR, Nutche J, Rosenberg DR, Keshavan M. Effect of antipsychotics on pituitary gland volume in treatment-naive first-episode schizophrenia: a pilot study. Schizophr Res. May 2007;92(1-3):207-210.

10. Takahashi T, Zhou SY, Nakamura K, et al. Longitudinal volume changes of the pituitary gland in patients with schizotypal disorder and first-episode schizophrenia. Prog Neuropsychopharmacol Biol Psychiatry. Jan 15 2011;35(1):177-183.

11. Nicolo JP, Berger GE, Garner BA, et al. The effect of atypical antipsychotics on pituitary gland volume in patients with first-episode psychosis: a longitudinal MRI study. Schizophr Res. Jan 2010;116(1):49-54.

12. Gruner P, Christian C, Robinson DG, et al. Pituitary volume in first-episode schizophrenia. Psychiatry Res. Jul 30 2012;203(1):100-102.

13. Riecher-Rössler A, Rybakowski J, Pflüger $M$, et al. Hyperprolactinemia in antipsychotic naïve patients with first episode psychosis - results from EUFEST. Psychol Med. 2013:DOI 10.1017/S0033291713000226.

14. Aston J, Rechsteiner E, Bull N, Borgwardt S, Gschwandtner U, Riecher-Rössler A. Hyperprolactinaemia in early psychosis-not only due to antipsychotics. Prog Neuropsychopharmacol Biol Psychiatry. Oct 1 2010;34(7):1342-1344.

15. Aiello G, Horowitz M, Hepgul N, Pariante CM, Mondelli V. Stress abnormalities in individuals at risk for psychosis: a review of studies in subjects with familial risk or with "at risk" mental state. Psychoneuroendocrinology. Oct 2012;37(10):1600-1613.

16. Habets $P$, Collip D, Myin-Germeys I, et al. Pituitary volume, stress reactivity and genetic risk for psychotic disorder. Psychol Med. Jul 2012;42(7):1523-1533.

17. Holtzman CW, Shapiro DI, Trotman HD, Walker EF. Stress and the prodromal phase of psychosis. Curr Pharm Des. 2012;18(4):527-533.

18. Riecher-Rössler A, Gschwandtner U, Aston J, et al. The Basel early-detection-of-psychosis (FEPSY)-study--design and preliminary results. Acta Psychiatr Scand. Feb 2007;115(2):114125. 
19. Gschwandtner U, Pflueger MO, Semenin V, Gaggiotti M, Riecher-Rössler A, Fuhr P. EEG: a helpful tool in the prediction of psychosis. Eur Arch Psychiatry Clin Neurosci. Aug 2009;259(5):257-262.

20. Lukoff D, Liberman RP, Nuechterlein KH. Symptom monitoring in the rehabilitation of schizophrenic patients. Schizophr Bull. 1986;12(4):578-602.

21. Riecher-Rössler A, Aston J, Ventura J, et al. Das Basel Screening Instrument fur Psychosen (BSIP): Entwicklung, Aufbau, Reliabilität und Validität. Fortschr Neurol Psychiatr. Apr 2008;76(4):207-216.

22. Woods SW. Chlorpromazine equivalent doses for the newer atypical antipsychotics. J Clin Psychiatry. Jun 2003;64(6):663-667.

23. Yung AR, Phillips LJ, McGorry PD, et al. Prediction of psychosis. A step towards indicated prevention of schizophrenia. Br J Psychiatry Suppl. 1998;172(33):14-20.

24. Yung AR, McGorry PD, Francey SM, et al. PACE: a specialised service for young people at risk of psychotic disorders. Med J Aust. Oct 1 2007;187(7 Suppl):S43-46.

25. Andreasen NC. The Scale for the Assessment of Negative Symptoms (SANS): conceptual and theoretical foundations. Br J Psychiatry Suppl. Nov 1989(7):49-58.

26. Buehlmann E, Berger GE, Aston J, et al. Hippocampus abnormalities in at risk mental states for psychosis? A cross-sectional high resolution region of interest magnetic resonance imaging study. J Psychiatr Res. May 2010;44(7):447-453.

27. Walter A, Studerus E, Smieskova R, et al. Hippocampal volume in subjects at high risk of psychosis: a longitudinal MRI study. Schizophr Res. Dec 2012;142(1-3):217-222.

28. Kappos L, Antel J, Comi G, et al. Oral fingolimod (FTY720) for relapsing multiple sclerosis. $N$ Engl J Med. Sep 14 2006;355(11):1124-1140.

29. Borgwardt SJ, McGuire PK, Aston J, et al. Structural brain abnormalities in individuals with an at-risk mental state who later develop psychosis. Br J Psychiatry Suppl. Dec 2007;51:s69-75.

30. Borgwardt SJ, Riecher-Rössler A, Dazzan P, et al. Regional gray matter volume abnormalities in the at risk mental state. Biol Psychiatry. May 15 2007;61(10):1148-1156.

31. Haller S, Borgwardt SJ, Schindler C, Aston J, Radue EW, Riecher-Rössler A. Can cortical thickness asymmetry analysis contribute to detection of at-risk mental state and first-episode psychosis? A pilot study. Radiology. Jan 2009;250(1):212-221.

32. R: A Language and Environment for Statistical Computing [computer program]. Vienna, Austria: R Foundation for Statistical Computing; 2012.

33. Baayen RH. languageR: Data sets and functions with "Analyzing Linguistic Data: A practical introduction to statistics". 2011.

34. Takahashi T, Nakamura K, Nishiyama S, et al. Increased pituitary volume in subjects at risk for psychosis and patients with first-episode schizophrenia. Psychiatry and clinical neurosciences. Nov 2013;67(7):540-548.

35. van Os J, Kenis G, Rutten BP. The environment and schizophrenia. Nature. Nov 11 2010;468(7321):203-212.

36. Phassouliotis C, Garner BA, Phillips LJ, et al. Enhanced cortisol suppression following administration of low-dose dexamethasone in first-episode psychosis patients. Aust $\mathrm{N} Z \mathrm{~J}$ Psychiatry. Nov 212012.

37. Borges S, Gayer-Anderson C, Mondelli V. A systematic review of the activity of the hypothalamic-pituitary-adrenal axis in first episode psychosis. Psychoneuroendocrinology. Jan 282013.

38. Phillips LJ, McGorry PD, Garner B, et al. Stress, the hippocampus and the hypothalamicpituitary-adrenal axis: implications for the development of psychotic disorders. Aust N Z J Psychiatry. Sep 2006;40(9):725-741.

39. Tognin S, Rambaldelli G, Perlini C, et al. Enlarged hypothalamic volumes in schizophrenia. Psychiatry Res. Nov 30 2012;204(2-3):75-81.

40. Mittal VA, Orr JM, Pelletier A, Dean DJ, Smith A, Lunsford-Avery J. Hypothalamic-pituitaryadrenal axis dysfunction in non-clinical psychosis. Psychiatry Res. Jan 162013. 
41. Romo-Nava F, Hoogenboom WS, Pelavin PE, et al. Pituitary volume in schizophrenia spectrum disorders. Schizophr Res. Mar 192013.

42. Belvederi Murri M, Pariante CM, Dazzan $\mathrm{P}$, et al. Hypothalamic-pituitary-adrenal axis and clinical symptoms in first-episode psychosis. Psychoneuroendocrinology. May 2012;37(5):629-644.

43. Krishnan KR, Doraiswamy PM, Lurie SN, et al. Pituitary size in depression. J Clin Endocrinol Metab. Feb 1991;72(2):256-259.

44. Axelson DA, Doraiswamy PM, Boyko OB, et al. In vivo assessment of pituitary volume with magnetic resonance imaging and systematic stereology: relationship to dexamethasone suppression test results in patients. Psychiatry Res. Oct 1992;44(1):63-70.

45. Takahashi T, Suzuki M, Velakoulis D, et al. Increased pituitary volume in schizophrenia spectrum disorders. Schizophr Res. Mar 2009;108(1-3):114-121.

46. Klomp A, Koolschijn PC, Hulshoff Pol HE, Kahn RS, Van Haren NE. Hypothalamus and pituitary volume in schizophrenia: a structural MRI study. Int J Neuropsychopharmacol. May 20 2011:1-8.

47. Tournikioti K, Tansella M, Perlini C, et al. Normal pituitary volumes in chronic schizophrenia. Psychiatry Res. Jan 15 2007;154(1):41-48.

48. Riecher-Rössler A, Hafner H. Schizophrenia and oestrogens--is there an association? Eur Arch Psychiatry Clin Neurosci. 1993;242(6):323-328.

49. Riecher-Rössler A, Häfner H, Dütsch-Strobel A, Stummbaum M. Gonadal function of schizophrenic and non-schizophrenic female inpatients. Arch Womens Ment Health. 1998;1:15-26.

50. Riecher-Rössler A. Oestrogen effects in schizophrenia and their potential therapeutic implications--review. Arch Womens Ment Health. Nov 2002;5(3):111-118.

51. Tsigkaropoulou E, Peppa M, Zompola C, et al. Hypogonadism due to hyperprolactinemia and subsequent first episode of psychosis. Gend Med. Feb 2012;9(1):56-60.

52. Lee BH, Kim YK. The relationship between prolactin response and clinical efficacy of risperidone in acute psychotic inpatients. Prog Neuropsychopharmacol Biol Psychiatry. Jun 2006;30(4):658-662.

53. Garcia-Rizo C, Fernandez-Egea E, Oliveira C, et al. Prolactin concentrations in newly diagnosed, antipsychotic-naive patients with nonaffective psychosis. Schizophr Res. Jan 2012;134(1):16-19.

54. Takahashi $T$, Suzuki $M$, Tanino $R$, et al. Volume reduction of the left planum temporale gray matter associated with long duration of untreated psychosis in schizophrenia: a preliminary report. Psychiatry Res. Apr 15 2007;154(3):209-219.

55. Mondelli V, Dazzan P, Gabilondo A, et al. Pituitary volume in unaffected relatives of patients with schizophrenia and bipolar disorder. Psychoneuroendocrinology. Aug 2008;33(7):10041012.

56. Elster AD. Modern imaging of the pituitary. Radiology. Apr 1993;187(1):1-14.

57. Suzuki M, Takashima T, Kadoya M, et al. Height of normal pituitary gland on MR imaging: age and sex differentiation. J Comput Assist Tomogr. Jan-Feb 1990;14(1):36-39.

58. Tsunoda A, Okuda O, Sato K. MR height of the pituitary gland as a function of age and sex: especially physiological hypertrophy in adolescence and in climacterium. AJNR Am J Neuroradiol. Mar 1997;18(3):551-554.

59. MacMaster FP, Keshavan M, Mirza Y, et al. Development and sexual dimorphism of the pituitary gland. Life Sci. Feb 13 2007;80(10):940-944.

60. Abech DD, Moratelli HB, Leite SC, Oliveira MC. Effects of estrogen replacement therapy on pituitary size, prolactin and thyroid-stimulating hormone concentrations in menopausal women. Gynecological endocrinology : the official journal of the International Society of Gynecological Endocrinology. Oct 2005;21(4):223-226.

61. Grams AE, Gempt J, Stahl A, Forschler A. Female pituitary size in relation to age and hormonal factors. Neuroendocrinology. 2010;92(2):128-132. 
62. Terano T, Seya A, Tamura Y, Yoshida S, Hirayama T. Characteristics of the pituitary gland in elderly subjects from magnetic resonance images: relationship to pituitary hormone secretion. Clin Endocrinol (Oxf). Sep 1996;45(3):273-279.

63. Habets $P$, Collip D, Myin-Germeys I, et al. Pituitary volume, stress reactivity and genetic risk for psychotic disorder. Psychol Med. Dec 1 2011:1-11.

64. Zipursky AR, Whittle $S$, Yucel $M$, et al. Pituitary volume prospectively predicts internalizing symptoms in adolescence. J Child Psychol Psychiatry. Mar 2011;52(3):315-323.

65. Ellis PD. The Essential Guide to Effect Sizes. Cambridge: Cambridge University Press; 2010. 